\title{
Modulating the Pattern Quality of Micropatterned Multilayer Films Prepared by Layer-by-Layer Self-Assembly
}

\author{
Jinhan Cho, Hongseok Jang, Bongjun Yeom, Hosub Kim, Raehyun Kim, Sangcheol Kim \\ and Kookheon Char* \\ School of Chemical and Biological Engineering \& NANO Systems Institute-National Core \\ Research Center, Seoul National University, \\ San 56-1, Shilim-dong, Kwanak-gu, Seoul 151-744, Korea \\ Frank Caruso \\ Centre for Nanoscience and Nanotechnology, Department of Chemical and Biomolecular \\ Engineering, The University of Melbourne, Victoria 3010, Australia
}

\section{Supporting Information}

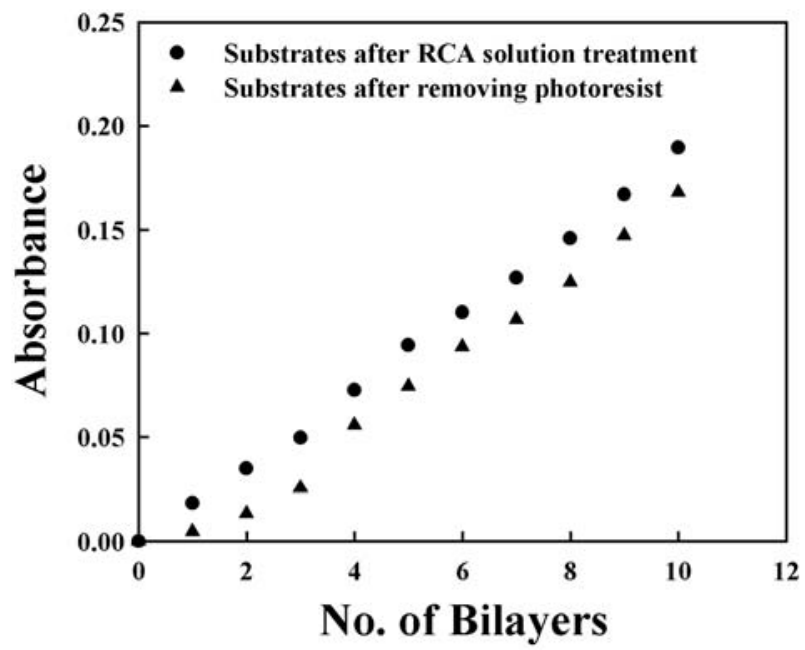

S1. UV-vis absorbance of (PAH/PSS) $)_{n}$ multilayer films deposited onto the substrates after RCA solution treatment $(\mathbf{O})$ and after removing the photoresists $(\boldsymbol{\Delta})$. The amount of added ionic salt into PE deposition solution was $0.4 \mathrm{M} \mathrm{NaCl}$. The RCA solution treatment, as detailed in references 44 and 46, involves negatively charging of silicon substrates by heating at $60{ }^{\circ} \mathrm{C}$ for $15 \mathrm{~min}$ in a 5:1:1 vol \% mixture of $\mathrm{H}_{2} \mathrm{O} / \mathrm{NH}_{3} / \mathrm{H}_{2} \mathrm{O}_{2}$. 


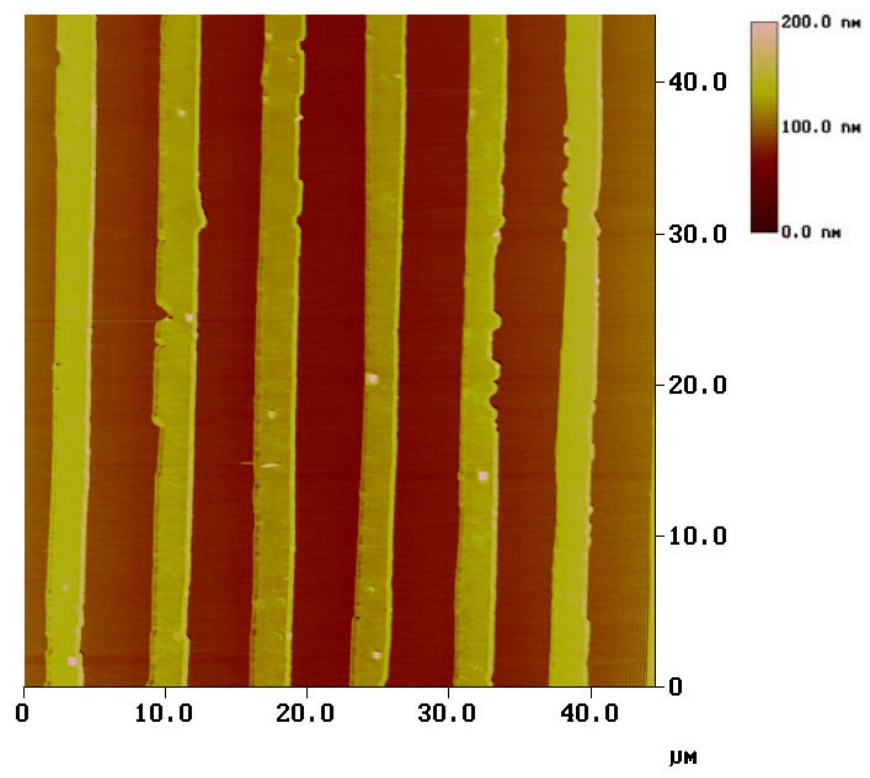

S2. AFM image of a patterned spin SALO multilayer films of (PAH/PSS) 20 prepared at $0.5 \mathrm{M} \mathrm{NaCl}$. 
(a)

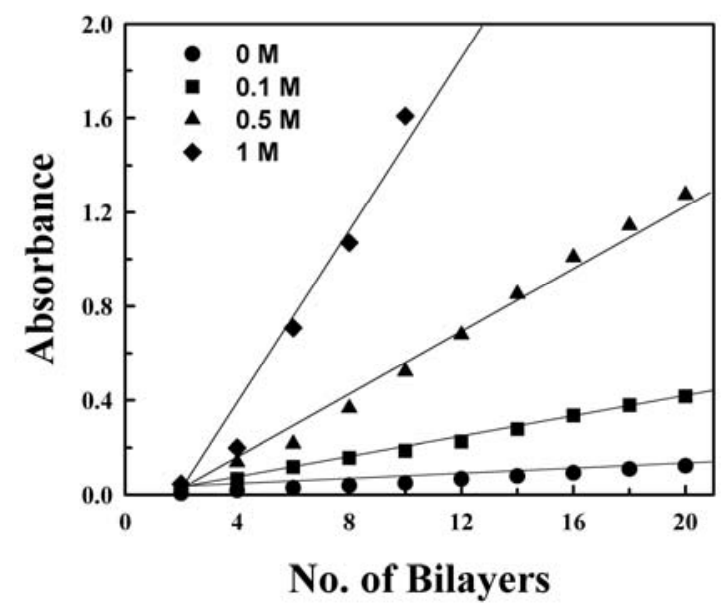

(b)

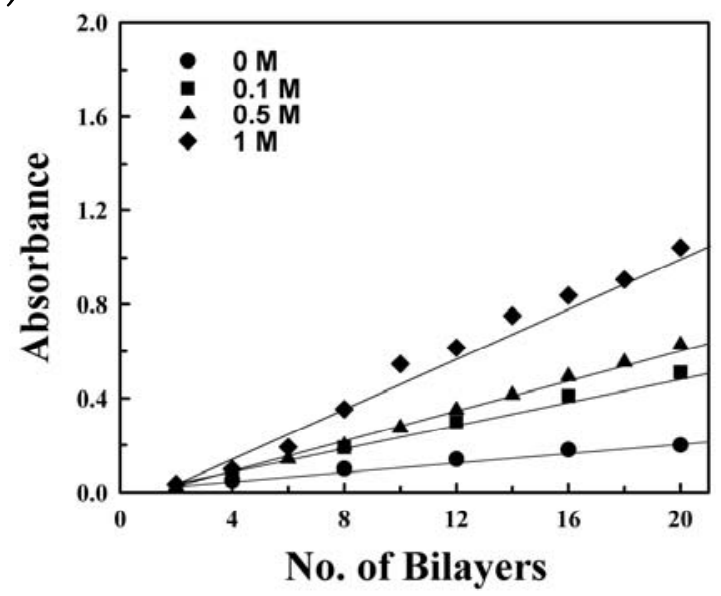

S3. Absorbance measured at $225 \mathrm{~nm}$ of (a) dip and (b) spin SA (PDADMAC/PSS) films with increasing the bilayer number at different salt concentrations. The absorbance values of dip SA films were divided by two for comparison with those of spin SA films, which are deposited only on one side of the substrate. These multilayer films were deposited onto planar quartz substrates without patterned photoresists. 